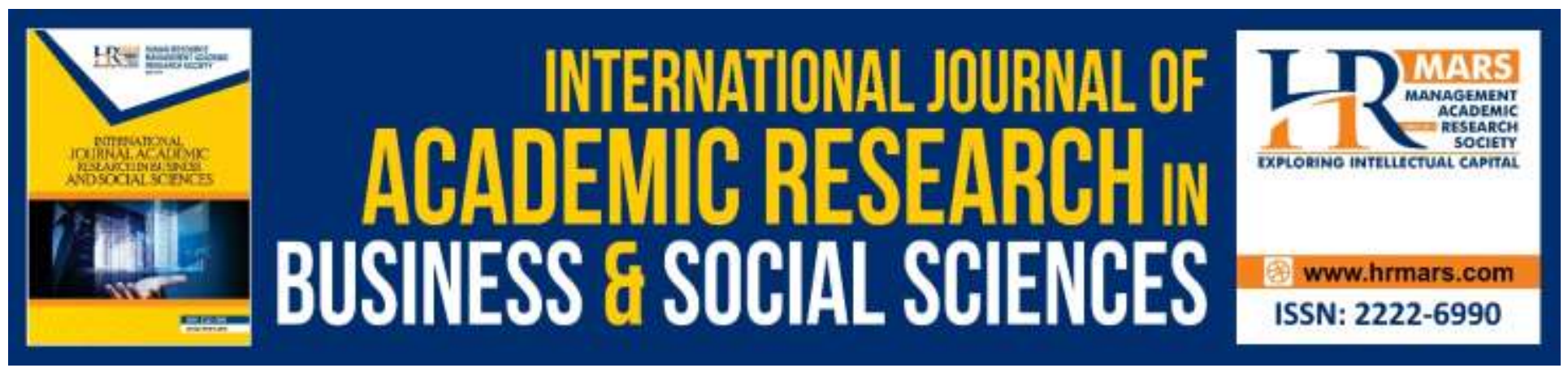

\title{
Novel Hybrid Ear Recognition Framework in Passive Human Identification
}

\author{
Ahmed Alemran, Bahbibi Rahmatullah
}

To Link this Article: http://dx.doi.org/10.6007/IJARBSS/v9-i14/6505

DOI:10.6007/IJARBSS/v9-i14/6505

Received: 22 June 2019, Revised: 17 July 2019, Accepted: 22 August 2019

Published Online: 02 October 2019

In-Text Citation: (Alemran \& Rahmatullah, 2019)

To Cite this Article: Alemran, A., \& Rahmatullah, B. (2019). Novel Hybrid Ear Recognition Framework in Passive Human Identification. International Journal of Academic Research in Business and Social Sciences, 9(14), 6370.

Copyright: (C) 2019 The Author(s)

Published by Human Resource Management Academic Research Society (www.hrmars.com)

This article is published under the Creative Commons Attribution (CC BY 4.0) license. Anyone may reproduce, distribute, translate and create derivative works of this article (for both commercial and non-commercial purposes), subject to full attribution to the original publication and authors. The full terms of this license may be seen

at: http://creativecommons.org/licences/by/4.0/legalcode

Vol. 9, No. 14, Special Issue: Education 4.0: Future Learning, Pg. 63 - 70

http://hrmars.com/index.php/pages/detail/IJARBSS

JOURNAL HOMEPAGE

Full Terms \& Conditions of access and use can be found at http://hrmars.com/index.php/pages/detail/publication-ethics 


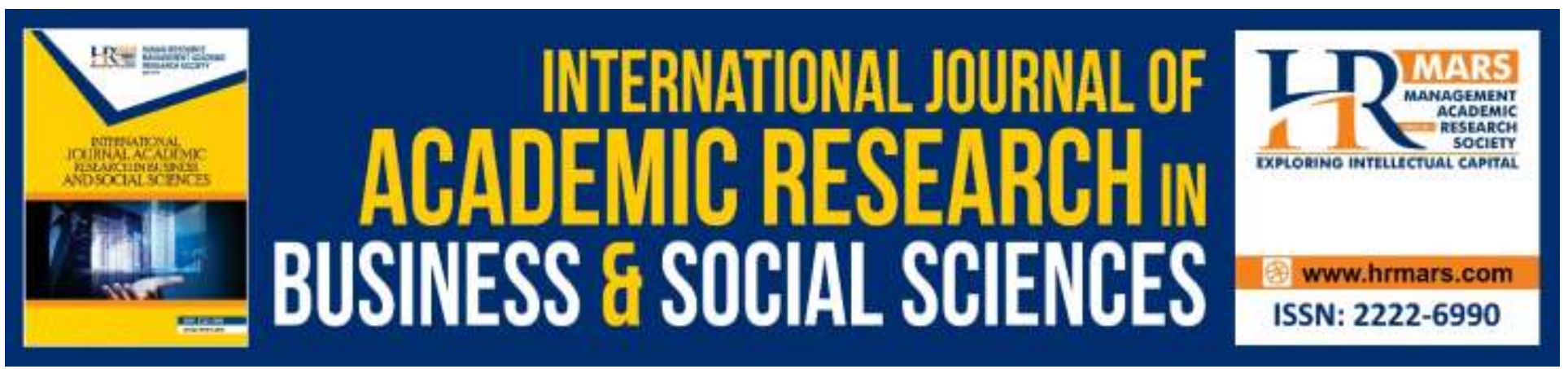

\title{
Novel Hybrid Ear Recognition Framework in Passive Human Identification
}

\author{
Ahmed Alemran, Bahbibi Rahmatullah \\ Department of Computing, Faculty of Arts, Computing and Creative Industry, University Pendidikan \\ Sultan Idris, Tanjong Malim, Perak, Malaysia \\ Email: ahmedalamran2@gmail.com, bahbibi@fskik.upsi.edu.my
}

\begin{abstract}
Human ear is an intriguing individual anatomical part of a passive, physiological biometrics systems based on the image acquired from digital cameras. The human ear has many singular features that permit the finding of particular individuals. It could be implemented as effective biometrics systems, for example, in crowd surveillance and identifying terrorist at public places such as airports, as well as controlling access to government offices.

Challenges and issues faced in the field of human identification using ear detection and recognition includes problem of occlusion, varieties in illumination, and real-time implementation in accessing information from an integrated database system with higher accuracy. Research on ear detection and recognition systems have been developing in a steady rate and mostly are constrained to controlled indoor environment. Notwithstanding, other research issues incorporate; ear print criminology; ear symmetry; and ear uniqueness.

This paper presents a review of existing biometric system based on ear features and proposes a novel hybrid ear recognition framework for the advancement of passive human identification technology. The aim of this work is to build a passive identification system for hybrid ear biometric from digital image database that is collected from two types of identifiers (right-left ear of the same person).

Keywords: Ear Biometrics, Ear Recognition, Ear Detection, Human Identification

\section{Introduction}

Biometric methods of individual identification have received much attention lately because it deals with important problems of human identification. In a passive biometrics system, important human anatomical parts captured from digital cameras showing faces and ears. Both of these body parts are important, as they allow for identifying many individuals. This can be applied for achieving efficient biometrics system. Nevertheless, there are many advantages in utilizing the ear as a source of data for an individual identification as it is smaller in size, stable in features and comes in colour images.
\end{abstract}


INTERNATIONAL JOURNAL OF ACADEMIC RESEARCH IN BUSINESS AND SOCIAL SCIENCES

Vol. 9, No. 14, Special Issue: Education 4.0: Future Learning. 2019, E-ISSN: 2222-6990 @ 2019 HRMARS

Furthermore, to enable the best listening, the ear is often not hidden underneath anything. (Choraś, 2005)

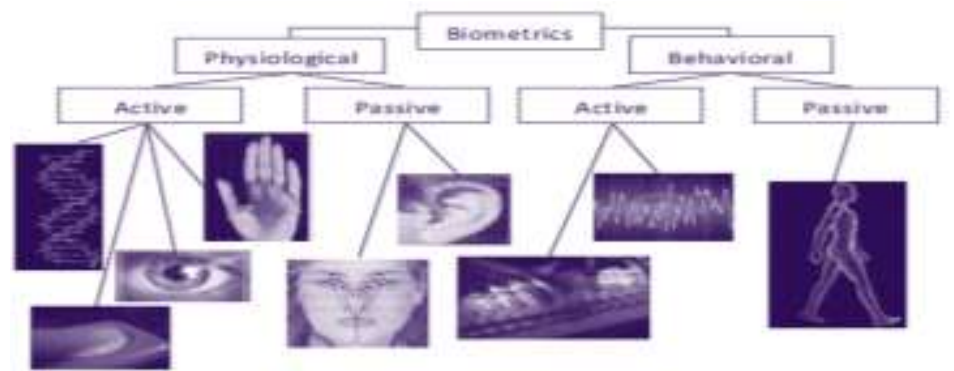

Figure 1 Biometrics' classes and technologies (Wahab, Hemayed, \& Fayek, 2012)

\section{Ear Biometric}

The term otomorphology, also commonly known as earology is the study of the physiognomy of the external ear for use in biometrics. Biometrics in light of the human ear is practical because the ear anatomy is unique for each individual and the elements for estimating the anatomy are comparable over time. The ear does not have a totally arbitrary structure; it is comprised of standard elements simply like the face. The parts of the human ear are minimal compared with the eyes, mouth, nose, and other facial elements. Natural human ear contains an external rim (helix) and ridges (anti-helix), concha, and tragus (small prominence of cartilage). Figure 2 shows parts of the anatomical elements (Omara et al. 2016)

1. Helix

2. Crus Antihelices

3. Antihelix

4. Scapha

5. Antitragus

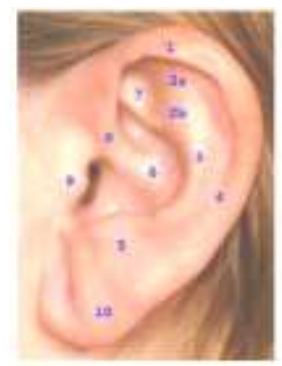

6. Cavum Conchae

7. Fossa Triangularis

8. Crus Helicis

Figure 2 Anatomy of the human ear (Omara et al., 2016)

We are focused on 2D ear image detection and recognition because standard digital cameras are well-established and less expensive than 3D scanners. 2D ear images are easily applicable in real-life access control applications. 2D ear images are more robust to problems such as occlusion while 3D ear images are more robust to illumination and pose (Chen, Mu, Nan, Zhang, \& Yang, 2017)

\section{Literature Review}

Many approaches have been proposed by several researchers to explain the topic of ear detection. According to Prakash (2008), automatic ear shape and size detection from side face could improve ear localization techniques. They tested their proposed technique on IIT Kanpur database with 95.2\% accuracy. In (Shih, Ho, Chang, \& Wu, 2009), a 2D ear detection method that utilized an edge-based algorithm is proposed to overcome the pose variation, rotation and illumination. Prakash et al. (2009) 
introduced an automatic ear localization in 2D side face images that only searches for ear in the skin areas. The method is proven to be faster to search on almost $60 \%$ of the area of the side face image. It was applied on IIT database including side face images of 150 persons and achieved an accuracy of 95.2\%. Prakash et al. (2009) introduced an efficient colour-skin and template based method for automatic detection of the ear in a side face image. The method first separates skin regions from non-skin regions before proceeding to look for the ear in skin regions. The process involves three phases; skin segmentation to eliminate all non-skin pixels from the ear image, ear localization to implement ear detection using template matching approach, and ear verification to support the ear detection using the Zernike moments based shape descriptor.

Prakash and Gupta (2012) introduced a new technique for rotation, scale, and shape invariant to localize the ear and utilizing the connected components in a graph obtained from the edge map of the side face image. They applied the proposed technique on IITK and UND databases with good accuracy.

Another interesting area in ear biometric system is the symmetric ear and segmentation. In (Xiaoxun \& Yunde, 2007), a new symmetrical null space algorithm with the eigenspaces was introduced and the findings show that Fisher features are more discriminative than eigenfeatures with respect to classification. Lu et al. (2006) applied Active Shape Model (ASM) to model the appearance of the ear image using a statistical method, and this method extracts features that can be used to improve the performance of the site format. Xiaoxun and Yunde (2007) suggested a novel ear and face recognition to symmetrical null space algorithm with an even-odd decomposition principle. Two orthogonal even-odd eigenspaces are constructed by using mirror images. Then, the characteristic features are extracted from the two eigenspaces under most situation of the null space. Finally, all the features are combined for classification task.

Alfred lannarelli who has served in numerous other law authority positions, has made two large-scale ear recognizable examinations in 1989 (Lammi, 2004). In the main research, there were more than ten thousand ears drawn from a randomly selected sample in California. The second research was for researching identical and non-identical twins. These researches support the hypothesis on ear uniqueness. Even the identical twins had comparable, but not identical, ear physiological components. He has created 12 measurements for identification, also known as "lannarelli System".

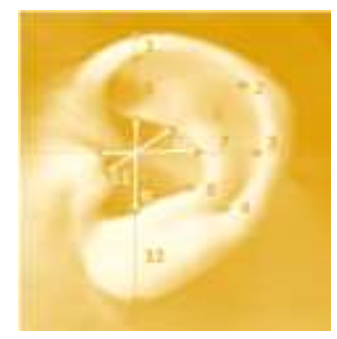

Figure 3: Iannarelli measurement (Abaza \& Ross, 2010) 
Burge and Burger (1998) presented the first automatic ear biometric method. They found ear biometrics to be promising because it is a robust and simple to be extracted biometric similar to fingerprinting. It is also suitable for passive identification, which is similar to face recognition. Choraś (2005) introduced a method to avoid the difficulty of localizing the anatomical points in lannarelli system. This method is based on the centre of the ear, which is the centre of the image itself. He proposed for a circle that has the same centre image in order to extract the features from the circle. The features are extracted by using two vectors; the first vector represents the characteristics of each circle, while the second vector represents the characteristics points for every contour in the normalized coordinates and treatment occlusion ear. Lu et al. (2006) proposed an ear shape features utilizing active shape model (ASM). Rich discriminate information of the local structural texture from the ear images are extracted and the eigen-ear shape technique was adopted for the classification. Left and right ear images are fusion to enhance the result. They collected 10 images from each individual; five for each side in 5 different poses from 56 volunteers. The recognition rate for the left ear image is $95.1 \%$ and the right is $93.3 \%$.

Anwar et al. (2015) suggested a new algorithm for ear recognition based on geometrical features extraction. Several values are extracted as feature vector; centre ( $X$ and $Y$ coordinates), and four different distances from the matrix that contain Euclidean distance between all pixels in the image. They tried to increase the number of distance values as to increase the feature vector that was represented. The experimental results give a result of $98 \%$ accuracy.

Texture and Depth Scale Invariant Feature Transform (TDSIFT) descriptor was introduced by Chen et al. (2017) in their ear recognition method. They combined the 2D and 3D data, and encode the 2D and 3D local features for ear recognition. The method tested on UND J2 database and achieved a recognition rate of $95.9 \%$ with average execution time of 9.33 seconds.
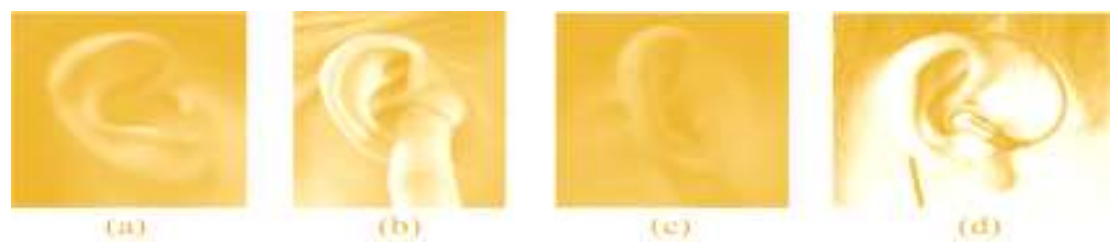

Figure 4 Examples of partial ear images (Chen \& Mu, 2016)

For the problem of ear classification stage and solving for occlusion, Burge and Burger (2000) proposed to utilize of thermogram ear images to detect most ear occlusion as hair, cap, etc. The temperature of hair is between $27.2^{\circ} \mathrm{C}$ and $29.7^{\circ} \mathrm{C}$, while the outer rim of the ear ranges from $30.0^{\circ} \mathrm{C}$ to $37.2^{\circ} \mathrm{C}$. The hair is removed by segmenting the lower temperature areas. B. Arbab-Zavar et al. (2007) utilized ear model to build an uncensored learning algorithm and a database purposely built for handling occlusion. This approach was successfully applied in XM2VTS database with a 91.5\% recognition rate. Yuan and $\mathrm{Mu}$ (2012) represented ear recognition system under variation of pose, lighting, and occlusion. The input is 2D ear image. The entire 2D image is separated into sub-images. Next, Neighbourhood Preserving Embedding is utilized for feature extraction on each sub-image. The most discriminative sub-images were selected as per the recognition rate. Each sub-image relates to 
INTERNATIONAL JOURNAL OF ACADEMIC RESEARCH IN BUSINESS AND SOCIAL SCIENCES

Vol. 9, No. 14, Special Issue: Education 4.0: Future Learning. 2019, E-ISSN: 2222-6990 @ 2019 HRMARS

a sub-classifier. Sub-classifiers are then merged for recognizing ear image with part occlusion. Chen and $\mathrm{Mu}$ (2016) proposed a weighted multi key point descriptor in sparse representation-based classification method that extracts local features of ear images. They utilized two databases; IIT and USTB. The average recognition time in each databases are 15.6 and 10.3 seconds. This method has considered the ear images that are affected by different occlusions.

\section{Proposed Framework}

Our proposed framework consists of three processes. The first of these is image acquisition process where we will utilize the IIT and USTB databases. The second process is designed to detect ear images from side profile face and applied skin segmentation and to calculate the edge map of the skin to create a suitable size of ear template. Ear localization from side face images will be done using Distance Transform and Template Matching followed by segmentation process to remove unwanted objects such as hat, hair or glasses frame from the image. The technique focuses on skin or non-skin segmentation, where the colour images will be converted to gray scale images in skin-likelihood image. Edge map of skin region will be computed and Canny edge detector is used to remove all edges that do not belong to the ear. Zernike moments are then applied to ensure this process (Surya Prakash, 2008). In this case we will take the ear image from various poses and angles, and we will apply on this image ASM to create a point-distributed model to normalize the ear image by calculating the longer axis from the outer contour. We will normalize ears for the longer axis to the same direction and length, then we will crop the ear with the border outer ear to get a pure image ear. Similarly for all previous stages we will detect the opposite ear to merge the left and right ear to create the proposed image (Figure $5(\mathrm{e})$ ).

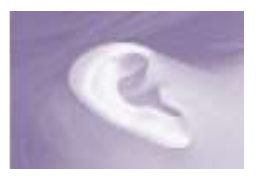

(a)

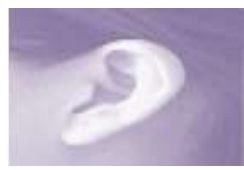

(b)

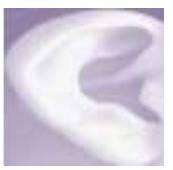

(c)

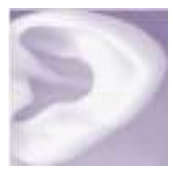

(d)

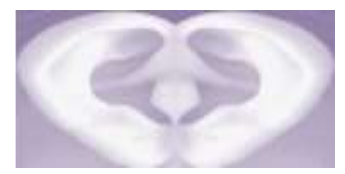

(e)

Figure 5 Proposed system (a) Right ear (b) Left ear (c) Right ear after normalization (d) Left ear after normalization (e) Merge ears to be proposed image (Mu, 2007)

In the third process, ear recognition will begin by dividing the proposed image to sub-images. Adjacent (sub-image) is obtained by shifting the previous sub-images by some pixels in a different direction. There will be an overlap in the adjacent sub-windows. The reason for this overlapping division is that it may remain some useful discriminating information on adjacent sub-images. Then each sub-image will be combined into a vector where all the vectors on the same locations from multiple ear images to form a sub-image set. Then the Gabor features of each sub-image are extracted and then their distance is to be less by Laplacian EigenMaps. The most discriminative subimage is selected using Sequential Forward Floating Selection. In this case, each column presents an ear image with its sub-images, while rows describe the sub-images detected on different ear images. 
INTERNATIONAL JOURNAL OF ACADEMIC RESEARCH IN BUSINESS AND SOCIAL SCIENCES

Vol. 9, No. 14, Special Issue: Education 4.0: Future Learning. 2019, E-ISSN: 2222-6990 @ 2019 HRMARS

So, not every sub-image is detected on every image. The frequency of detection varies for different sub-images (Arbab-Zavar and Nixon, 2011).

In feature extraction, Neighbourhood Preserving Embedding is applied to get the projection matrix for each sub-space. With the projection matrix, the dimensionality of ear image will be reduced and its feature vector will be extracted. We will use Neighbourhood Preserving Embedding for sub-image ranking, where each sub-image region forms a sub-classifier. So we will design a score level fusibility for multiple sub-classifiers. The top classification sub-classifiers are selected for the fusibility. The weighted sum is utilized for the result level fusibility; the more distinguished sub-classifier gets a higher weight. Before the fusibility, the match scores of each sub-classifier are transformed into a common domain using the z-score normalization (Yuan \& Mu, 2012). We are planning to apply our proposed system by choosing three images from each ear sides making a total of six images for each person and arrange it sequentially $(1 R, 1 L)(1 R, 2 L)(1 R, 3 L)(2 R, 1 L) \ldots(3 R, 3 L)$ which will give us nine sets of images per person. We propose to use the USTB database and the IIT Delhi database.

\section{Conclusion}

The main goal of this paper is to review related literatures in ear identification and to develop a system capable of interactively making decision in the human ear detection and identification with lower running time and complexity. So the proposed framework for detection introduces a highly efficient algorithm in ear images processing based on a new feature extraction method that combines the local and global data in various poses of ear images. The proposed techniques will be tested on USTB databases and IIT Delhi Database because it contains suitable images.

\section{References}

Abaza, A., \& Ross, A. (2010). Towards understanding the symmetry of human ears: A biometric perspective. IEEE 4th International Conference on Biometrics: Theory, Applications and Systems, BTAS 2010. https://doi.org/10.1109/BTAS.2010.5634535

Alemran, A., \& Rahmatullah, B. (2019). Novel Hybrid Ear Recognition Framework in Passive Human Identification. International Journal of Academic Research in Business and Social Sciences, 9(14), 1-8.

Anwar, A. S., Ghany, K. K. A., \& Elmahdy, H. (2015). Human Ear Recognition Using Geometrical Features Extraction. Procedia Computer Science, 65(Iccmit), 529-537. https://doi.org/10.1016/j.procs.2015.09.126

Arbab-Zavar, B., Nixon, M. S., \& Hurley, D. J. (2007). On Model-Based Analysis of Ear Biometrics. 2007 First IEEE International Conference on Biometrics: Theory, Applications, and Systems. https://doi.org/10.1109/BTAS.2007.4401937

Arbab-Zavar, Banafshe, \& Nixon, M. S. (2011). On guided model-based analysis for ear biometrics. Computer Vision and Image Understanding, 115(4), 487-502.

https://doi.org/10.1016/j.cviu.2010.11.014

Burge, M., \& Burger, W. (1998). Using Ear Biometrics for Passive Identification. 14th IIternational Conference on Information Security, 98, 139-148.

Burge, M., \& Burger, W. (2000). Ear biometrics in computer vision. Proceedings 15th International Conference on Pattern Recognition. Los Alarnitos, CA, USA: IEEE Computer Society., 2, 822-826. 
INTERNATIONAL JOURNAL OF ACADEMIC RESEARCH IN BUSINESS AND SOCIAL SCIENCES

Vol. 9, No. 14, Special Issue: Education 4.0: Future Learning. 2019, E-ISSN: 2222-6990 @ 2019 HRMARS

https://doi.org/10.1109/ICPR.2000.906202

Chen, L., \& Mu, Z. (2016). Partial Data Ear Recognition From One Sample per Person Long. leee Transactions on Human-Machine Systems, 1-11.

Chen, L., Mu, Z., Nan, B., Zhang, Y., \& Yang, R. (2017). TDSIFT: a new descriptor for 2D and 3D ear recognition. Eighth International Conference on Graphic and Image Processing (ICGIP 2016), 10225(Icgip 2016), 102250C. https://doi.org/10.1117/12.2266727

Choraś, M. (2005). Ear Biometrics Based on Geometrical Feature Extraction. Electronic Letters on Computer Vision and Image Analysis, 5(3), 84-95. https://doi.org/10.1007/978-3-540-300748_7

Lammi, H. (2004). Ear biometrics. Tech. Rep. Lappeenranta University of Technology., 1-6.

Lu, L., Xiaoxun, Z., Youdong, Z., \& Yunde, J. (2006). Ear Recognition Based on Statistical Shape Model. First International Conference on Innovative Computing, Information and Control Volume I (ICICIC'06), 3. https://doi.org/10.1109/ICICIC.2006.445

$\mathrm{Mu}$, Z. (2007). Ear Recognition based on 2D Images. 2007 First IEEE International Conference on Biometrics: Theory, Applications, and Systems, (1), 1-5. https://doi.org/10.1109/BTAS.2007.4401941

Omara, I., Li, F., Zhang, H., \& Zuo, W. (2016). A novel geometric feature extraction method for ear recognition. Expert Systems with Applications, 65, 127-135. https://doi.org/10.1016/j.eswa.2016.08.035

Prakash, S., \& Gupta, P. (2012). An efficient ear localization technique. Image and Vision Computing, 30(1), 38-50. https://doi.org/10.1016/j.imavis.2011.11.005

Prakash, S., Prakash, S., Jayaraman, U., Jayaraman, U., Gupta, P., \& Gupta, P. (2009). A Skin-Color and Template Based Technique for Automatic Ear Detection. Icapr, 213-216. https://doi.org/10.1109/ICAPR.2009.31

Saranya, M., Cyril, G. L. I., \& Santhosh, R. R. (2016). An approach towards ear feature extraction for human identification. International Conference on Electrical, Electronics, and Optimization Techniques, ICEEOT 2016, 4824-4828. https://doi.org/10.1109/ICEEOT.2016.7755636

Shih, H. C., Ho, C. C., Chang, H. T., \& Wu, C. S. (2009). Ear detection based on arc-masking extraction and AdaBoost polling verification. IIH-MSP 2009 - 2009 5th International Conference on Intelligent Information Hiding and Multimedia Signal Processing, 669-672. https://doi.org/10.1109/IIH-MSP.2009.75

Surya Prakash, U. J. and P. G. (2008). Ear Localizatilon from Side Face images using Distance Transform and Template Matching. Image (Rochester, N.Y.), (c).

Wahab, N. K. A., Hemayed, E. E., \& Fayek, M. B. (2012). HEARD: An automatic human EAR detection technique. International Conference on Engineering and Technology, ICET 2012 - Conference Booklet. https://doi.org/10.1109/ICEngTechnol.2012.6396118

Xiaoxun, Z., \& Yunde, J. (2007). Symmetrical null space LDA for face and ear recognition. Neurocomputing, 70(4-6), 842-848. https://doi.org/10.1016/j.neucom.2006.10.016

Yuan, L., \& Mu, Z. C. (2012). Ear recognition based on local information fusion. Pattern Recognition Letters, 33(2), 182-190. https://doi.org/10.1016/j.patrec.2011.09.041 